ZERTIFIZIERTE FORTBILDUNG

IN ZUSAMMENARBEIT MIT DER BAYERISCHEN LANDESÄRZTEKAMMER

Folge 136

Hier können Sie CME-Punkte sammeln a) für die Pflichtfortbildung aller Vertragsärzte und

b) für freiwillige Fortbildungszertifikate, die viele Landesärztekammern anbieten.

Die Multiple-Choice-Fragen beziehen sich auf den vorangegangenen Fortbildungsbeitrag (Seiten 27-31). Die Antworten ergeben sich z. T. aus dem Text, z. T. beruhen sie auf medizinischem Basiswissen!

Wenn Sie 70\% der Fragen richtig beantworten, erhalten Sie 2, bei 100\% 3 CME-Punkte.

Einsendeschluss: 20. Januar 2008 (www.cme-punkt.de) bzw. 4. Oktober 2007 (Teilnahme per Brief).
MWW

Fortschritte der Medizin

CME-Herausgeber- und Review-Board:

Dr. H. J. K. Barwitz, Prof. Dr. A. Berghaus, Prof. Dr. Dr. h.c. Th. Brandt, Prof. Dr. W. G. Daniel, Prof. Dr. W. Eisenmenger, Prof. Dr. K. Friese, Prof. Dr. H. S. Füessl, Prof. Dr. B. Göke, Prof. Dr. R. Gradinger, Prof. Dr. Dr. h.c. A. Hofstetter, Prof. Dr. H. Holzgreve, Prof. Dr. A. Imdahl, Prof. Dr. K. Krüger, Prof. Dr. H.-J. Möller, Prof. Dr. Dr. h.c. G. Plewig, Prof. Dr. D. Reinhardt, U. Weigeldt.

\title{
Fragen zum Thema „Harninkontinenz der Frau“
}

\section{- 1. Welche Therapie gehört nicht zu den konservativen Maßnahmen bei der \\ Belastungsharninkontinenz?}

A Physiotherapie

B Beckenbodentraining

C Elektrostimulation

D Lokale Östrogentherapie

E Tension-free Vaginal Tape

\section{- 2. Welche Wirkung kommt bei der} Elektrostimulationstherapie nicht vor?

A Kontraktion der quer gestreiften Beckenbodenmuskulatur

B Muskelhypertrophie

C Muskeltonisierung

D Muskelvaporisation

E Muskelhyperplasie

\section{- 3. Was bedeutet Biofeedback-Becken- bodentraining?}

A Kontraktion der quer gestreiften Beckenbodenmuskulatur

B Umsetzung der Muskelaktivität beim kontrollierten Beckenbodentraining in ein akustisches oder visuelles Signal

C Relaxation der Beckenbodenmuskulatur

C Toiletten- und Miktionstraining

D Konditionierungstraining

\section{- 4. Welcher Effekt wird durch Östrogene im unteren Harntrakt nicht erzielt?}

A Proliferationssteigerung der Epithel von Scheide, Vulva und Urethra

B Adrenerger Einfluss

C Anticholinerger Einfluss

D Endokriner Einfluss

E Cholinerger Einfluss

\section{- 5. Nennen Sie einen Wirkstoff für die medikamentöse Behandlung der \\ Belastungsharninkontinenz.}

A Tolterodin

B Duloxetin

C Darifenacin

D Trospiumchlorid

E Propiverinhydrochlorid

\section{- 6. Welche Antwort trifft zu?}

A Östrogenovula haben nur lokale Effekte.

B Reizblasensyndrome sind eine Kontraindikation gegen die lokale Östrogentherapie.

C Östrogenovula können zur Antikonzeption verwendet werden.

D Östrogene fördern die Proliferation des Vaginal- und Harnröhrenurothels.

E Östrogene haben keinen Einfluss auf cholinerge Rezeptoren des Urogenitaltrakts.

\section{- 7. Die Inzidenz von Harninkontinenz und} Genitaldeszensus ist nicht erhöht bei

A Zwillingsgeburten.

B Multiparität.

C Herzinsuffizienz.

D chron. Obstipation mit Hämorrhoiden.

E Frauen, die schwer körperlich arbeiten.

\section{- 8. Die Integraltheorie von Petros und Ulmsten}

A versucht eine Drangsymptomatik mit Inkontinenz anhand einer Lockerheit des perivaginalen Bandapparats zu erklären.

B sieht in der Beckenbodengymnastik zur Stärkung der einzelnen Muskelgruppen eine Möglichkeit, defekte Bandstrukturen auszugleichen.
C konstatiert, dass Kontinenz nur durch einen suffizienten Schließmuskel erreicht wird.

D erklärt neurogene Blasenentleerugsstörungen.

E kann nur bei Patientinnen ohne Hystektomie die Inkontinenzsymptomatik erklären.

\section{- 9. Eine Dranginkontinenz}

A kann niemals durch eine akute Infektion bedingt sein.

B kann nur durch aufwendige Untersuchungen wie z.B. dem dynamischem MRT diagnostiziert werden.

C tritt nur bei älteren Frauen mit Östrogenmangel auf.

D ist in der Ätiologie einfach zu erfassen.

E tritt häufig auch als Leitsymptom mit Mikro-und Makrohämaturie bei Blasentumor auf.

\section{- 10. Die vaginale Untersuchung mit simulierter Operation}

A ersetzt die gynäkologische Vorsorgeuntersuchung.

B zeigt durch den Pinchtest, ob eine Korrektur des hinteren Kompartiments eine Verbesserung der Drangsymptomatik ewarten lässt.

C hat nur bei Patientinnen nach Hysterektomie eine Aussagekraft.

D ist auf der Grundlage der Integraltheorie ein gutes Instrument, um den operativen Erfolg einer Beckenbodenrekonstruktion abzuschätzen.

E kann keine Aussage zum Therapieerfolg bei mehrfach voroperierten Patientinnen treffen.

\section{ANTWORTFORMULAR}

Mit Einreichen dieses Fragebogens erkläre ich mich damit einverstanden, dass die angegebenen Daten zum Zweck der Teilnahmebestätigung gespeichert und bei erfolgreicher Teilnahme an den Einheitlichen Informationsverteiler (EIV) der Ärztekammern weitergegeben werden.

Wichtiger Hinweis:

Eine Auswertung ist künftig nur noch möglich, wenn Sie Ihre EFN auf dem Antwortformular angeben! Nähere Hinweise hierzu unter: www.cme-punkt.de/faq.html
„Harninkontinenz der Frau“

MMW-Fortschr. Med. Nr. 38 / 2007 (149. Jg.)

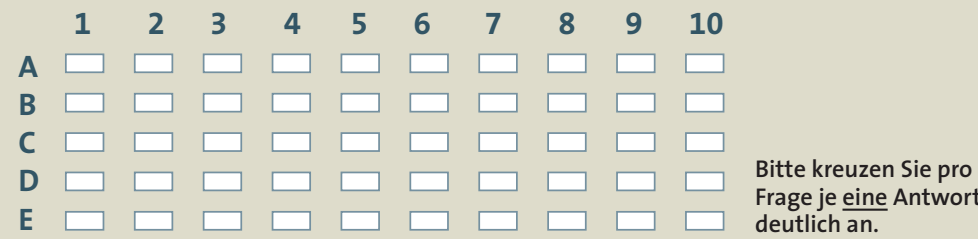

Name, Vorname

Fachrichtung

Geburtsdatum

Einheitliche Fortbildungsnummer (EFN)
Und so kommen Sie zu Ihren Punkten:

Teilnahme im Internet: unter www.cme-punkt.de. Dort führen wir für Sie ein elektronisches Punktekonto.

Teilnahme per Brief: Fragebogen ausfüllen und mit frankiertem Rückumschlag an: Urban \& Vogel GmbH, Redaktion MMW-Fortschr. Med. Abt. Fortbildungszertifikat, Neumarkter Str. 43 , 81673 München. 